\title{
openheart Heart failure: a story of damage, fatigue and injury?
}

\author{
Prithwish Banerjee $e^{1,2,3}$
}

To cite: Banerjee P. Heart failure: a story of damage, fatigue and injury?. Open Heart 2017;4:e000684. doi:10.1136/ openhrt-2017-000684

Received 10 July 2017 Revised 29 August 2017 Accepted 26 September 2017

\section{CrossMark}

${ }^{1}$ Department of Cardiology, University Hospitals Coventry and Warwickshire, Coventry, UK ${ }^{2}$ Faculty of Health and Life Sciences, Coventry University, Coventry, UK

${ }^{3}$ Warwick Medical School, University of Warwick, Coventry, UK

Correspondence to Prof Prithwish Banerjee; Prithwish.Banerjee@uhcw. nhs.uk

\section{ABSTRACT}

Heart failure has been recognised for years but the complete picture has been difficult to clearly understand. This article aims to try and put forward a proposed mechanistic explanation to encompass all that we see within the clinical heart failure syndrome using supporting published evidence. The aim of the article is to link, using published evidence, all the known varieties of heart failure into a spectrum that is explained by simple interlinked processes. In addition, the concept of routinely looking for reversibility of left ventricular dysfunction is introduced.

\section{TRADITIONAL UNDERSTANDING OF HEART FAILURE}

Historically, heart failure was thought to be a disorder of its pumping function and the ejection fraction $(\mathrm{EF})$; therefore, these became an important component and determinant of the condition. The increasing prevalence of heart failure with preserved ejection fraction (HFPEF) and the current lack of disease-modifying therapy for this group of patients raise the question again of whether we truly understand the mechanisms that drive the entire spectrum of the heart failure syndrome. How can all the different described types of heart failure be linked up to create a spectrum and a story that is easily understandable? Can we link the relationship of the core mechanism of heart failure with $\mathrm{EF}$ and then with the types of HF such as heart failure with reduced ejection fraction (HFREF), HFPEF or even with the old classification of high-output, low-output, forward and backward failure, right and left heart failure? Unless we progress further in answering these questions, discovering treatment for the entire heart failure population will be difficult.

\section{Damage, fatigue and injury concept}

For chronic heart failure, there may be three basic but linked mechanisms that exist to cause the heart failure: damage, fatigue and injury.

The damage mechanism is very evident in the common myocardial infarctions ${ }^{1}$ or some toxic/inflammatory cardiomyopathies (eg, anthracycline chemotherapy-induced cardiomyopathy or chronic myocarditis) ${ }^{2}$ and is naturally associated with reduced EF (HFREF). Here, there is a large amount of irreversible myocardial damage leading subsequently to fibrosis; the heart failure is systolic and is due to pump failure.

However, myocardial injury can often be transient, acute and limited, such as with acute viral myocarditis causing myocardial inflammation and oedema, or other reversible cardiomyopathies (injury of an undefined nature in peripartum cardiomyopathy, a sympathoexcitation-induced injury in Takotsubo cardiomyopathy or a hypermetabolic injury in heart failure related to a thyroid storm). ${ }^{3}$ It would not be unreasonable to assume that the reversible forms of left ventricular (LV) systolic dysfunction are the ones where injury has played a part but significant damage has not occurred (the injury group). There could be variable amounts of injury and damage caused by alcohol, chemotherapeutic agents or other drugs, catecholamines, infections and so on with the final outcome on LV function determined by the proportion of injury versus damage. Genetic causes or myocardial infiltration would most likely also fall under the injury versus damage headings.

Myocardial fatigue may be the cause of heart failure if the ventricle is having to pump against high vascular resistance. ${ }^{45}$ This may be the main mechanism of $\mathrm{HF}$ in patients with HFPEF as there is increasing evidence that microvascular dysfunction ${ }^{67}$ is probably the key operating mechanism in this variety of heart failure. Data have been presented to suggest that patients with HFPEF are likely to be progressing from a stage of preclinical diastolic dysfunction to the HFPEF stage with increasing left ventricular end-diastolic pressure (LVEDP) as assessed by echocardiographic $\mathrm{E} / \mathrm{E}^{\prime} .{ }^{8}$ It can be speculated that the elevated LVEDP is related to the LV struggling to pump into a high-pressure vascular circuit that is a result of microvascular dysfunction. Ageing ${ }^{9-11}$ has an important role in gradually increasing arterial resistance as 
have comorbidities ${ }^{12}$ such as hypertension ${ }^{13}$, diabetes ${ }^{14} 15$ and chronic kidney disease. ${ }^{16}$ There is probably additional contribution to this vascular ageing from changes in the heart itself of both microvascular rarefaction and myocardial fibrosis (including perivascular fibrosis), which reduces coronary flow reserve resulting in $\mathrm{LV}$ diastolic dysfunction. ${ }^{18}$

Cardiac fatigue has been described in athletes, ${ }^{19}{ }^{20}$ is reversible and appears to affect ventricular diastolic function $^{21}$ more than systolic function. The above hypothesis is, therefore, plausible. While fatigue in the early stages would manifest as increased LVEDP with preserved LV systolic function, prolonged or severe fatigue would naturally lead to systolic dysfunction and pump failure (which may be reversible if the fatigue can be tackled by say controlling poorly controlled blood pressure ${ }^{22}$ ) probably because of the development of focal and diffuse myocardial fibrosis that accompanies severe fatigue. Systemic hypertension, the the most common and most important cause of HFPEF, causes myocardial fatigue as a result of the enhanced vascular stiffness against which the LV has to pump. Another parallel example of fatigue would be HF related to conditions such as hypertrophic obstructive cardiomyopathy $(\mathrm{HOCM})^{23}$ and severe aortic stenosis, ${ }^{24}$ which increase LVEDP by causing intramyocardial resistance to blood flow at the level of the left ventricular outflow tract and the stenotic aortic valve, respectively. In these conditions, the heart failure is usually of the HFPEF type as the ventricle is having to pump against high resistance (which is at the cardiac rather than at the vascular level), with a consequent increase in LVEDP. Interestingly, intervention to reduce the high resistance, such as an aortic valve replacement for severe aortic stenosis, can result in recovery of the $\mathrm{LV}$ fatigue ${ }^{24}$ particularly in those without myocardial fibrosis, ${ }^{25}$ presumably as the myocardium in these patients is in a state of chronic fatigue that has not reached an advanced stage that is accompanied by fibrosis. On the other hand, advanced and prolonged fatigue can result in irreversible myocardial changes of hypertrophy and fibrosis ${ }^{26}$ as seen in some patients with severe untackled aortic stenosis with no cardiac reserve. In other words, recovery is linked to the stage of fatigue. Advanced fatigue with extensive fibrosis is unlikely to recover.

Advanced myocardial fibrosis that is seen in both patients with aortic stenosis (AS) ${ }^{27}$ and patients with $\mathrm{HOCM}^{28}$ may be a consequence of chronic progressive fatigue through myocyte loss, but in HOCM, myocardial abnormalities including hypertrophy and fibrosis also have a genetic basis. In non-obstructive hypertrophic cardiomyopathy, the hypertrophy and fibrosis are genetically determined and lead to a purely cardiac cause of HFPEF due to LV diastolic dysfunction with little contribution from vascular stiffness.

Looking at the phenotypes of AS from a fatigue angle, AS with preserved LVEF would correspond to early LV fatigue with preserved $\mathrm{EF}$ and stroke volume at the expense of elevated LVEDP. Later when LVEDP rises substantially, LV filling becomes impaired leading to low-flow, low-gradient AS with preserved LVEF, but the stroke volume and cardiac output would have reduced. Finally, as advanced fatigue sets in, there is fibrosis due to myocyte loss, LVEF starts to fall and the ventricle starts to dilate leading ultimately to a dilated ventricle with reduced EF, the so-called low-flow, low-gradient severe AS with depressed EF. A ventricle suffering from uncorrected advanced fatigue can, therefore, end up looking like one that is a result of damage. The pathophysiology of the end stage, however, is likely to be different in the fatigued ventricle with focal and diffuse fibrosis occurring in a chronic slow fashion as opposed to fairly rapid fibrosis that occurs due to damage during infarction or inflammation.

High-output cardiac failure ${ }^{29}$ would also be an example of fatigue-related heart failure as a result of the myocardium's inability to cope with an enhanced volume of blood.

\section{WHERE DOES HEART FAILURE WITH MID-RANGE EJECTION FRACTION FIT INTO THIS?}

The European Society of Cardiology has introduced the new category of heart failure with mid-range ejection fraction (HFMREF) in its new classification in $2016^{30}$ defined as signs and symptoms of heart failure with LVEF between $40 \%$ and $49 \%$. It has been suggested that HFMREF is a unique phenotype of heart failure, and studies on this group have shown mortality and readmission rates between that seen in HFREF and HFPEF patients. ${ }^{31}$ Sticking with the same hypothesis that HFREF is mainly a myocardial problem that is damage related and HFPEF mainly a vascular problem that is fatigue related, HFMREF may be HFPEF that is gradually dropping LVEF due to prolonged or increasing fatigue. In other words, HFMREF may be simply another fatigue-related vascular issue but at a more advanced level than HFPEF. Of course, two other components of HFMREF would be patients with HFREF who have suffered mild damage or patients with HFREF who originally had severe damage but have now improved their EF. These subsets were assessed in a recent study ${ }^{32}$ in which the demographics of the HFMREF cohort were found to be heterogeneous, with more coronary artery disease in the HFMREF improved EF group (improved from below $\mathrm{EF}<40 \%$ ) and more hypertension and diastolic dysfunction in the HFMREF deteriorated EF group (deteriorated from $\mathrm{EF}>50 \%$ ). The other two groups compared were HFREF and HFPEF. HFMREF improved EF patients had significantly $(p<0.001)$ better clinical outcomes relative to matched patients with HF and reduced ejection fraction, and significantly $(\mathrm{p}<0.01)$ improved clinical outcomes relative to HFMREF deteriorated patients, whereas clinical outcomes of the HFMREF deteriorated subgroup of patients were not significantly different from matched patients with HF with preserved ejection fraction. These results appear to suggest that the patients with HFMREF that are HFREF showing improvement of EF do better than patients with HFPEF with reducing EF. 


\section{ASSESSMENT OF ARTERIAL STIFFNESS}

Of a number of non-invasive methods that are available to assess vascular stiffness, the two commonly used ones are pulse wave velocity (PWV) and augmentation index (AI) ${ }^{33}$ The elasticity of a segmental artery (say carotidfemoral) is assessed by PWV. A pulse wave is generated by each cardiac contraction, which travels distally and can be measured by the distance travelled by the pulse wave divided by the time taken between two regions such as carotid to femoral. More the arterial stiffness, more the PWV. Carotid-femoral PWV measures central arterial stiffness, ${ }^{34}$ whereas brachial-ankle PWV measures both central and peripheral arterial stiffness. ${ }^{35}$ As changes in blood pressure (BP) can affect these measurements, a new cardio-ankle vascular index $(\mathrm{CAVI})^{36}$ has been used that is independent of BP. CAVI uses measured PWV adjusted by a stiffness index $\beta$. Particularly in the elderly, arterial stiffness independently predicts cardiovascular morbidity and mortality. ${ }^{37-39}$

AI is derived from the interaction between the forward pulse wave and the reflected wave and is a measure of resistance of the entire arterial tree. ${ }^{40}$ It increases with increasing arterial stiffness ${ }^{41}$ and is influenced both by $\mathrm{BP}^{33}$ and heart rate. ${ }^{42}$ Although both PWV and AI increase with age, changes in AI are more prominent in younger people below $<50$ years of age and PWV changes are more marked in those older than 50 years ${ }^{43}$. In women after menopause, arterial resistance rises sharply and is higher than in men. ${ }^{445}$

A review of the literature on vascular stiffness does support the hypothesis that ageing-related arterial stiffness ${ }^{46}$ coupled with comorbidities such as hypertension ${ }^{47}$ and diabetes, ${ }^{48}$ which enhance the stiffness, can lead to HFPEF. Support of this also comes from a small non-invasive study comparing arterial compliance, venous capacitance and microvascular function in three patients' groups comprising HFPEF, HFREF and control (non-HF). The study found that there was significantly reduced vascular compliance (increased arterial stiffness, decreased venous capacitance) in the HFPEF group compared with the HFREF and control groups $(\mathrm{p}<0.05) .{ }^{49}$

\section{HOW MIGHT MECHANISTIC THINKING IN RELATION TO INVESTIGATIONS FOR HF HELP?}

Thinking of damage, fatigue or injury while investigating someone with heart failure may be clinically useful to try and predict if the heart failure may be reversible in an individual and thereby link to prognosis in that individual. A clinician may identify the aetiological factors that might predict the degree of injury versus damage on a cardiac MRI scan and make a better judgement of the long-term outcome. A good example would be the degree of myocardial oedema versus patchy scarring seen on a cardiac MRI scan (CMR) in a patient with myocarditis, which helps to assess just this. A heavily scarred LV from a large myocardial infarct seen on CMR is quickly judged as having had a large amount of damage that would not be reversible. On the other hand, the finding of elevated LVEDP on echocardiography with preserved LVEF along with elevated brain natriuretic peptide but with no infarction or infiltration on cardiac MRI (and normal perfusion) could point the finger at fatigue-related HFPEF, which can be potentially reversed if a reversible precipitating factor (such as poorly controlled blood pressure or diabetes) can be identified or new research is able to find a cure for microvascular dysfunction. In the case of HFMREF, the finding of significant infarction could separate out the damage-related HFMREF (where HFREF drugs should still work) from the fatigue-related HFMREF (where HFREF drugs are unlikely to work). Mechanistic thinking would also help with understanding why the EF is preserved, low or mid range in a patient with heart failure. For example, a CMR showing reduced LVEF with no infarction but reduced perfusion would be able to explain the HFREF by a mechanism of fatigue (muscle weakness) but due to reduced perfusion to the cardiac muscle rather than fatigue secondary to elevated arterial resistance.

\section{ASSESSING FOR REVERSIBILITY OF LV DYSFUNCTION}

While assessing a patient with heart failure, an important aim of a heart failure physician is to identify whether the heart failure may be due to a reversible process as identification of reversible factors may lead to effective treatment of these and subsequent complete cure of the HF. Looking for underlying aetiologies and precipitating factors forms part of this assessment. The assessment could be further aided by linking in the mechanism of the heart failure (damage, fatigue or injury) with evidence gathered from available investigations such as a cardiac MRI scan, myocardial perfusion scan, dobutamine stress echo and so on. Focal replacement fibrosis, corresponding to areas of myocyte loss, is seen well by delayed gadolinium enhancement on CMR and linked with irreversible impairment of $\mathrm{LV}$ systolic function and a worse prognosis. ${ }^{5051}$ A novel method to detect a different type of fibrosis- diffuse interstitial myocardial fibrosis, using T1 mapping by CMR, ${ }^{52}$ has also now been developed and will aid this process of assessment for reversible LV dysfunction. While diffuse fibrosis by T1 mapping is also a marker of worse prognosis, it may not be as concerning as focal fibrosis and there is current interest in it as a therapeutic tool ${ }^{54}$. T1 mapping is likely to become a really well-established tool for cardiac disease assessment ${ }^{55}$ in the near future. The common reversible cardiomyopathies $^{56}$ (tachycardia induced, peripartum, inflammatory/ infectious, hyperthyroidism induced, Takotsubo and chronic illness induced such as with cirrhosis, obesity, uraemia) could all be mechanistically assessed in this manner to further enhance our expected prediction of the degree of reversibility of LV systolic function in individual cases. Knowledge on the degree of reversibility of LV dysfunction is likely to improve decision making on treatment options (for example, a large amount of 


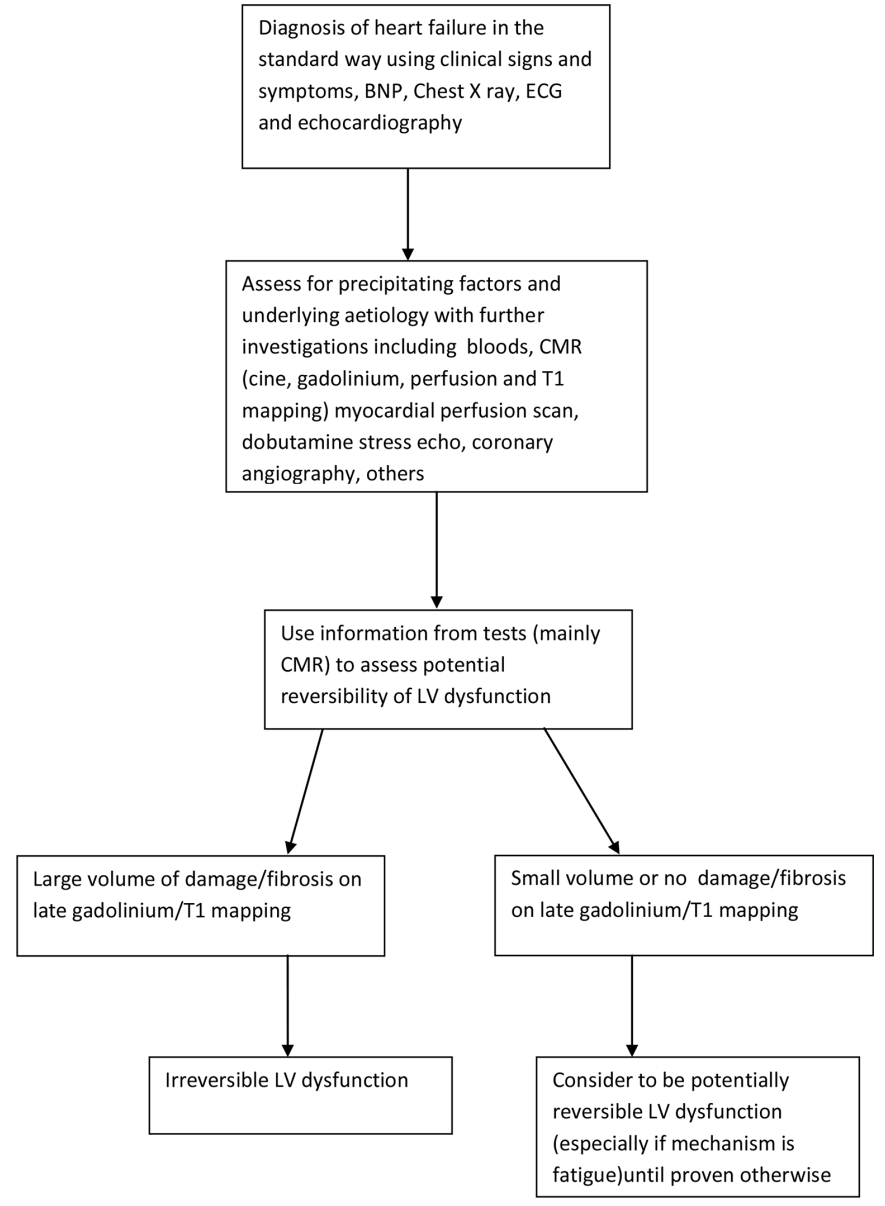

Figure 1 Suggested plan of assessment of reversibility of LV dysfunction in a patient with heart failure. Although not perfect by any means, this is a starting point. BNP, brain natriuretic peptide; CMR, cardiac MRI scan; LV, left ventricle.

expected reversibility should point towards intensive care treatment in the acute setting and consideration for mechanical circulatory support rather than palliative care in the appropriate patient). A proposed pathway of assessment of heart failure to discern reversibility is provided in figure 1 as a starting point in this exercise.

\section{CONCLUSION}

Systolic or diastolic dysfunction (or ejection fraction) may not be as important as the underlying mechanism of damage, fatigue or injury in the heart failure syndrome in understanding the core mechanism of heart failure in an individual patient. There will often be a mixture of systolic and diastolic dysfunctions in many patients with a predominance of diastolic impairment in the fatigue variety and of systolic dysfunction in the damage variety. As understanding of the entire heart failure syndrome improves, the classification may change to reduce its reliance on ejection fraction and may contain new elements such as arterial resistance, ventricular end-diastolic pressure and perhaps even basic mechanistic concepts such as damage, fatigue and injury. Further research focusing particularly on myocardial fatigue in patients with HFPEF will determine whether this mechanistic model of damage, fatigue and injury truly stands up to scrutiny.

Contributors The entire article is conceived and written by PB alone.

Competing interests None declared.

Provenance and peer review Not commissioned; externally peer reviewed.

Data sharing statement No additional data available.

Open Access This is an Open Access article distributed in accordance with the Creative Commons Attribution Non Commercial (CC BY-NC 4.0) license, which permits others to distribute, remix, adapt, build upon this work non-commercially, and license their derivative works on different terms, provided the original work is properly cited and the use is non-commercial. See: http://creativecommons.org/ licenses/by-nc/4.0/

(C) Article author(s) (or their employer(s) unless otherwise stated in the text of the article) 2017. All rights reserved. No commercial use is permitted unless otherwise expressly granted.

\section{REFERENCES}

1. Cleland JG, McGowan J. Heart failure due to ischaemic heart disease: epidemiology, pathophysiology and progression. $J$ Cardiovasc Pharmacol 1999;33(suppl 3):S17-29.

2. Ghuran A, Nolan J. Recreational drug misuse: issues for the cardiologist. Heart 2000;83:627-33.

3. Huffman C, Wagman G, Fudim M, et al. Reversible cardiomyopathies - a review. Transplant Proc 2010;42:3673-8.

4. Balmain S, Padmanabhan N, Ferrell WR, et al. Differences in arterial compliance, microvascular function and venous capacitance between patients with heart failure and either preserved or reduced left ventricular systolic function. Eur J Heart Fail 2007;9:865-71.

5. Maréchaux S, Samson R, van Belle E, et al. Vascular and microvascular endothelial function in heart failure with preserved ejection fraction. J Card Fail 2016;22:3-11.

6. Lee JF, Barrett-O'Keefe Z, Garten RS, et al. Evidence of microvascular dysfunction in heart failure with preserved ejection fraction. Heart 2016;102:278-84.

7. Giamouzis G, Schelbert EB, Butler J. Growing evidence linking microvascular dysfunction with heart failure with preserved ejection fraction. J Am Heart Assoc 2016;5:e003259.

8. Banerjee P, Motiwala A, Mustafa HM, et al. Does left ventricular diastolic dysfunction progress through stages? insights from a community heart failure study. Int J Cardiol 2016;221:850-4.

9. Izzo JL, Shykoff BE. Arterial stiffness: clinical relevance, measurement, and treatment. Rev Cardiovasc Med 2001;2:297-3440.

10. Taddei S, Virdis A, Ghiadoni L, et al. Age-related reduction of NO availability and oxidative stress in humans. Hypertension 2001;38:274-9.

11. Csiszar A, Wang M, Lakatta EG, et al. Inflammation and endothelial dysfunction during aging: role of NF-kappaB. J Appl Physiol 2008;105:1333-41.

12. Paulus WJ, Tschöpe C. A novel paradigm for heart failure with preserved ejection fraction: comorbidities drive myocardial dysfunction and remodeling through coronary microvascular endothelial inflammation. J Am Coll Cardiol 2013;62.

13. Melenovsky V, Borlaug BA, Rosen B, et al. Cardiovascular features of heart failure with preserved ejection fraction versus nonfailing hypertensive left ventricular hypertrophy in the urban Baltimore community: the role of atrial remodeling/dysfunction. J Am Coll Cardiol 2007;49:198-207.

14. Schram MT, Henry RM, van Dijk RA, et al. Increased central artery stiffness in impaired glucose metabolism and type 2 diabetes: the Hoorn Study. Hypertension 2004;43:176-81.

15. Owan TE, Hodge DO, Herges RM, et al. Trends in prevalence and outcome of heart failure with preserved ejection fraction. $N$ Engl $J$ Med 2006;355:251-9.

16. Wang MC, Tsai WC, Chen JY, et al. Arterial stiffness correlated with cardiac remodelling in patients with chronic kidney disease. Nephrology 2007;12:591-7.

17. Edwards NC, Steeds RP, Stewart PM, et al. Effect of spironolactone on left ventricular mass and aortic stiffness in early-stage chronic kidney disease: a randomized controlled trial. J Am Coll Cardiol 2009;54:505-12. 
18. Schwartzkopff B, Brehm M, Mundhenke M, et al. Repair of coronary arterioles after treatment with perindopril in hypertensive heart disease. Hypertension 2000;36:220-5.

19. Douglas PS, O'Toole ML, Hiller WD, et al. Cardiac fatigue after prolonged exercise. Circulation 1987;76:1206-13.

20. Oxborough D, Birch K, Shave R, et al. "Exercise-induced cardiac fatigue" - a review of the echocardiographic literature. Echocardiography 2010;27:1130-40

21. Kasikcioglu E. Diastolic performance after prolonged exercise. Med Sci Sports Exerc 2005;37:164.

22. Cioffi G, Stefenelli C, Tarantini L, et al. Chronic left ventricular failure in the community: prevalence, prognosis, and predictors of the complete clinical recovery with return of cardiac size and function to normal in patients undergoing optimal therapy. J Card Fail 2004:10:250-7.

23. Sanderson JE, Gibson DG, Brown DJ, et al. Left ventricular filling in hypertrophic cardiomyopathy. an angiographic study. Br Heart $J$ 1977;39:661-70.

24. Villari B, Vassalli G, Monrad ES, et al. Normalization of diastolic dysfunction in aortic stenosis late after valve replacement. Circulation 1995;91:2353-8.

25. Barone-Rochette G, Piérard S, De Meester de Ravenstein C, et al Prognostic significance of LGE by CMR in aortic stenosis patients undergoing valve replacement. J Am Coll Cardiol 2014;64:144-54.

26. Milano AD, Faggian G, Dodonov M, et al. Prognostic value of myocardial fibrosis in patients with severe aortic valve stenosis. J Thorac Cardiovasc Surg 2012;144:830-7.

27. Dweck MR, Joshi S, Murigu T, et al. Midwall fibrosis is an independent predictor of mortality in patients with aortic stenosis. J Am Coll Cardiol 2011;58:1271-9.

28. Galati G, Leone O, Pasquale F, et al. Histological and histometric characterization of myocardial fibrosis in end-stage hypertrophic cardiomyopathy: a clinical-pathological study of 30 explanted hearts. Circ Heart Fail 2016;9:e003090.

29. Anand IS, Florea VG. High output cardiac failure. Curr Treat Options Cardiovasc Med 2001;3:151-9.

30. Ponikowski P, Voors AA, Anker SD, et al. 2016 ESC Guidelines for the diagnosis and treatment of acute and chronic heart failure. Eur Heart J 2016;37:2129-200.

31. Pascual-Figal DA, Ferrero-Gregori A, Gomez-Otero I, et al. Midrange left ventricular ejection fraction: clinical profile and cause of death in ambulatory patients with chronic heart failure. Int J Cardiol 2017;240:265-70.

32. Rastogi A, Novak E, Platts AE, et al. Epidemiology, pathophysiology and clinical outcomes for heart failure patients with a mid-range ejection fraction. Eur J Heart Fail 2017;17.

33. Tomiyama $\mathrm{H}$, Yamashina $\mathrm{A}$. Non-invasive vascular function tests: their pathophysiological background and clinical application. Circ $J$ 2010;74:24-33.

34. Mancia G, De Backer G, Dominiczak A, et al. 2007 guidelines for the management of arterial hypertension: the task force for the management of arterial hypertension of the European Society of Hypertension (ESH) and of the European Society of Cardiology (ESC). J Hypertens 2007;25:1105-87.

35. Yamashina A, Tomiyama H, Takeda K, et al. Validity, reproducibility, and clinical significance of noninvasive brachial-ankle pulse wave velocity measurement. Hypertens Res 2002;25:359-64.

36. Kubozono T, Miyata M, Ueyama K, et al. Clinical significance and reproducibility of new arterial distensibility index. Circ $J$ 2007;71:89-94.

37. Meaume S, Benetos A, Henry OF, et al. Aortic pulse wave velocity predicts cardiovascular mortality in subjects $>70$ years of age. Arterioscler Thromb Vasc Biol 2001:21:2046-50.
38. Mattace-Raso FU, van der Cammen TJ, Hofman A, et al. Arterial stiffness and risk of coronary heart disease and stroke: the Rotterdam Study. Circulation 2006;113:657-63.

39. Matsuoka O, Otsuka K, Murakami S, et al. Arterial stiffness independently predicts cardiovascular events in an elderly community - Longitudinal Investigation for the Longevity and Aging in Hokkaido County (LILAC) study. Biomed Pharmacother 2005;59(Suppl 1):S40-4

40. Nichols WW. Clinical measurement of arterial stiffness obtained from noninvasive pressure waveforms. Am J Hypertens 2005;18:3-10.

41. Wilkinson IB, MacCallum H, Hupperetz PC, et al. Changes in the derived central pressure waveform and pulse pressure in response to angiotensin II and noradrenaline in man. J Physiol 2001;530:541-50.

42. Williams B, Lacy PS. CAFE and the ASCOT (Anglo-Scandinavian Cardiac Outcomes Trial) Investigators. Impact of heart rate on central aortic pressures and hemodynamics: analysis from the CAFE (Conduit Artery Function Evaluation) study: CAFE-Heart Rate. J Am Coll Cardiol 2009;54:705-13.

43. McEniery CM, Yasmin HIR, Qasem A, et al. Normal vascular aging: differential effects on wave reflection and aortic pulse wave velocity: the Anglo-Cardiff Collaborative Trial (ACCT). J Am Coll Cardiol 1760;2005:1753-60.

44. Kim JY, Park JB, Kim DS, et al. Gender difference in arterial stiffness in a multicenter cross-sectional study: the Korean Arterial Aging Study (KAAS). Pulse 2014;2:11-7.

45. Vermeersch SJ, Rietzschel ER, De Buyzere ML, et al. Age and gender related patterns in carotid-femoral PWV and carotid and femoral stiffness in a large healthy, middle-aged population. $J$ Hypertens 2008;26:1411-9.

46. Lee HY, Oh BH. Aging and arterial stiffness. Circ J 2010;74:2257-62.

47. Amar J, Ruidavets JB, Chamontin B, et al. Arterial stiffness and cardiovascular risk factors in a population-based study. $J$ Hypertens 2001;19:381-7.

48. Scuteri A, Najjar SS, Muller DC, et al. Metabolic syndrome amplifies the age-associated increases in vascular thickness and stiffness. J Am Coll Cardiol 2004:43:1388-95.

49. Balmain S, Padmanabhan N, Ferrell WR, et al. Differences in arterial compliance, microvascular function and venous capacitance between patients with heart failure and either preserved or reduced left ventricular systolic function. Eur J Heart Fail 2007;9:865-71.

50. Mewton N, Liu CY, Croisille P, et al. Assessment of myocardial fibrosis with cardiovascular magnetic resonance. J Am Coll Cardiol 2011;57:891-903.

51. McCrohon JA, Moon JC, Prasad SK, et al. Differentiation of heart failure related to dilated cardiomyopathy and coronary artery disease using gadolinium-enhanced cardiovascular magnetic resonance. Circulation 2003;108:54-9.

52. Pattanayak P, Bluemke DA. Cardiac MR imaging to probe tissue composition of the heart by using T1 mapping. Radiology 2014;271:320-2.

53. Tham EB, Haykowsky MJ, Chow K, et al. Diffuse myocardia fibrosis by T1-mapping in children with subclinical anthracycline cardiotoxicity: relationship to exercise capacity, cumulative dose and remodeling. J Cardiovasc Magn Reson 2013;15:48.

54. Stuckey DJ, McSweeney SJ, Thin MZ, et al. T $\square$ mapping detects pharmacological retardation of diffuse cardiac fibrosis in mouse pressure-overload hypertrophy. Circ Cardiovasc Imaging 2014;7:240-9.

55. Moon JC, Treibel TA, Schelbert EB. T mapping for diffuse myocardial fibrosis. J Am Coll Cardiol 2013;62:1288-9.

56. Patel H, Madanieh R, Kosmas CE, et al. Reversible Cardiomyopathies. Clin Med Insights Cardiol 2015;9(Suppl 2):7-14. 